\title{
Study on Diffusion Coefficient of Fluorophores in 3D Hydrogel with Cationic Charge using Microchip
}

\author{
Ju Young Jin ${ }^{1, \#, ~ J a e s o o l ~ S h i m ~}{ }^{2, \#}$, and Jinseok Kim ${ }^{1, *}$ \\ ${ }^{1}$ Center for Bionics, Biomedical Research Institute, Korea Institute of Science and \\ Technology, Hwarangno 14-gil 5, Seongbuk-gu, Seoul 136-791, Republic of Korea \\ ${ }^{2}$ School of Mechanical Engineering, Yeungnam University, Gyeongsan, Gyeonsanbuk-do \\ 712-749, Republic of Korea
}

\begin{abstract}
Author Information
\# Ju Young Jin and Jaesool Shim contributed equally to this work.

*Corresponding Authors. E-mail: jinseok@kist.re.kr
\end{abstract}

\begin{abstract}
The diffusion coefficients of ions are measured in a microchip filled with a cationic charged 3D hydrogel in order to study the effect of cationic charged 3D hydrogel on the diffusivity of ions. In this study, poly-diallyl-dimethyl-ammoniumchloride (poly-DADMAC) is used to produce a 3D hydrogel. Four different fluorophores are used in the 3D hydrogel rhodamine 6G, rhodamine-BSA, fluorescein isothio-cyanate (FITC) and FITC-BSA. The rhodamine $6 \mathrm{G}$ and rhodamine-BSA are positively charged (cations), while fluorescein isothio-cyanate (FITC) and FITC-BSA are negatively charged (anions). Two widely used techniques which are short time diffusivity measurement technique and long time diffusivity measurement techniques are used to measure the diffusion coefficients. For the short time measurement, Fluorescence recovery after photo-bleaching (FRAP) is used by a 3D confocal microscope. For the long time measurement, fluorescence images are taken for 11 days to observe a pure diffusivity without any convective movement. As a result, the diffusivity of the cations was found to be lower than that of the anions in the cationic charged hydrogel.
\end{abstract}


Keywords - Fluorescence recovery after photo bleaching (FRAP), Diffusion coefficient, electric charge, Poly-DADMAC, 3D hydrogel

\section{Introduction}

Hydrogels, also well known as aquagels, are composed of a three-dimensional network of hydrophilic polymer chains. Hydrogels can be used to deliver soluble or immobilized signals to the cells, act as support structures for cell growth and function, and provide space filling for the future tissue ingrowth. It is highly absorbent and therefore can swell or shrink by absorbing or emitting water [1-2]. Hydrogels are commonly used as scaffolds in tissue engineering [3-4] and as biosensors that respond to certain molecules such as glucose or antigens [5]. In addition, they have been used to release drugs in drug delivery systems [6-7].

These key features of hydrogels such as swelling and shrinking under water have been rapidly studied as potential materials for various biomedical applications [8-11]. In many cases, hydrogels have been applied as a 'stealth material' since it is inert to most biological molecules such as proteins. Various molecules can be encapsulated in the hydrogels. Since hydrogel molecules are inert and do not bind with most chemicals, drugs or other proteins, a variety of molecules can be released from hydrogels based on the molecular diffusion rate as well as the pore diameter of the polymetric network. Diffusivity in hydrogels is of significant importance in mimicking the actual biological environment. Besides molecular weight or size, diffusivity can be affected greatly by the polarity or electric charge density of the bio molecules and hydrogels. 
There are a variety of studies on measurement and analysis of diffusivity of molecules in hydrogels for biomedical applications.

In 1985, Lee examined both experimentally and theoretically the kinetics of drug release from hydrogel matrices. He found that the release of water soluble drugs from initially dehydrated hydrogel matrices generally involves the simultaneous absorption of water and desorption of drug through a swelling-controlled diffusion mechanism [12]. In 1993, Merrill et al. experimentally determined the ratio of diffusion coefficient in hydrogels of poly ethylene oxide(PEO) and diffusion coefficient in free solution. They found the effective diffusion coefficients of oxygen, hydrogen peroxide and hydroquinone in a hydrogel under steady state conditions. In their study, the diffusion rate for the materials was independent of the degree of cross-linking of the hydrogel [13]. In 1996, Li et al. determined diffusion coefficients for relevant solutes [ranging in size from oxygen to immunoglobulin G (IgG)] into and out of agarose and alginate gels. They show that the determination is highly reproducible and useful for gels crosslinked in the cylindrical geometry in order for studying transport through matrices used in cell immobilization [14]. In 1997, Matsuyama et al. examined diffusion and portioning of solutes which have various molecular weights in poly(vinyl alcohol) hydrogel membrane. The solute diffusion through swollen gel membrane was analyzed by the equation based on free volume theory and the efficiency of the proposed analysis equation was confirmed by the experimental results of the effects of solute size and water volume fraction in the membrane [15]. In 2006, Liang et al. conducted the determination of proteins' diffusion in agarose hydrogel at body temperature using their insitu refractive index method. They found that the diffusion coefficients of proteins decrease with the increase of the concentration of agarose and the solute molecular size [16]. In 2009, Koutsopoulosa et al. showed that the release kinetics for a variety of proteins of wide range 
of molecular mass, hydrodynamic radii, and isoelectric points through a nanofiber hydrogel scaffold consisting of designer self-assembling peptides by using single-molecule fluorescence correlation spectroscopy (FCS). Their results showed that this biocompatible and injectable designer self-assembling peptide hydrogel system may be useful as a carrier for therapeutic proteins for sustained release applications [17]. In 2010, Park et al. presented an approach to generate microporous cell-laden hydrogels for fabricating biomimetic tissueengineered constructs, and characterized the physical, mechanical, and biological properties (i.e., microporosity, diffusivity, and cell viability) of cell-laden agarose gels as a function of engineered porosity. In their study, microporosity within agarose gels can also control diffusion profiles that significantly affect cell viability in the medium perfusion condition [18]. Recently, in 2015, Lopez-Sanchez et al. studied diffusion of macromolecules in selfassembled cellulose/hemicellulose hydrogels using fluorescence recovery after photobleaching (FRAP). They investigated the diffusivities of a series of fluorescently labelled dextrans of different molecular weight, and proteins including a plant pectin methyl esterase (PME) [19]. In the same year, Patra et al. showed the Diffusion of Moisture from Hydrogel Scaffold with Induced Porosity from Self-Assembled Bubbles. In their study a mathematical model was used to derive an effective diffusivity of the scaffold as a function of the moisture content [20].

Although a variety of experiments on diffusivity in various hydrogels are conducted, the diffusivity has been mainly determined in terms of the mechanical properties of hydrogels such as molecular weight or size, mass and pore size of the matrix as well as porosity of hydrogels. 
However, in many cases, since there are electric interactions between the molecules and the matrix of hydrogels under specific charge conditions, the electric forces hinder diffusivity of molecules from hydrogels or enhance diffusivity of them. we experimentally found that the diffusivity of molecules is also highly dependent on the charge conditions of both molecules and hydrogels. In this study, the diffusion coefficients of charged molecules are measured in a cationic charged 3D hydrogel by using microchips in order to study the interaction effect of charge conditions of hydrogels.

Diffusion coefficients of fluorophores with electric charges in poly-diallyl-dimethylammoniumchloride (poly-DADMAC) hydrogels have been used to measure diffusivity of charged molecules.

Fluorescence recovery after photo-bleaching that has been widely used to quantify the two dimensional lateral diffusion of fluorescent molecules was utilized to measure the diffusion coefficients of fluorophores. In addition, a long-term diffusivity test was also conducted to compare the results that were obtained by FRAP.

\section{Experimental Methods}

\section{Microchannel fabrication}

The procedure for the fabrication of the microchannels used for the measurement of diffusion coefficient is based on a photo lithography method widely used to make PDMS microchips. Briefly, for a pattern replica, the SU-8 2035 as a negative photoresist is coated on a silicon-wafer substrate using a spin coater. The spin rate was controlled and programmed according to the processing guidelines of MICROCHEM for the desired channel thickness and coating quality [21]. For the PDMS channel, the PDMS pre-polymer and curing agent 
(Sylgard 184; Dow Corning, Midland, MI, USA) were uniformly mixed at a ratio of 10:1, and degassed to remove bubbles inside the PDMS mixture. The PDMS mixture formed on a master silicon substrate was cured in the oven. After the curing process, the cured PDMS was carefully peeled off from the Si-wafer substrate. The PDMS channel had holes as reservoirs at the end of the channels. The PDMS with a channel pattern and a slide glass for the bottom of the channel were bonded by treating it with the plasma cleaner.

Figure 1 shows a schematic and a picture of the fabricated microchannel. The height of the channel is $25 \mu \mathrm{m}$ and the total height of the PDMS mold is $0.8 \mathrm{~mm}$ for this experiment.

\section{Poly-DADMAC synthesis}

A poly-DADMAC solution was synthesized by using $4.8 \mathrm{~mL}$ of poly(acrylamide-codiallyl-dimethyl-ammoniumchloride) solution $\left(\right.$ Sigma $\left.^{\circledR}\right), 0.05 \mathrm{~g}$ of $\mathrm{N}, \mathrm{N}^{\prime}$-Methylenebis (acrylamide; Sigma ${ }^{\circledR}$ ) that acted as a cross-linker, and $0.05 \mathrm{~g}$ of 2-Hydroxy-4'-(2hydroxyethoxy)-2-methylpropiophenone $\left(\right.$ Sigma $\left.^{\circledR}\right)$ that acted as a photoinitiator. The synthesized poly-DADMAC solution was injected into the microchannel and cured by using ultraviolet light (UV). Omnicure ${ }^{\circledR}$ S2000 was used as an ultraviolet light source and polyDADMAC solution was cured for $10 \mathrm{~s}$ using $20 \mathrm{~W}$ of power.

\section{Fluorescence synthesis}

Four fluorescent materials were used to measure the diffusivities of molecules with different electric charges: rhodamine 6G $\left(\right.$ Sigma $\left.^{\circledR}\right)$, rhodamine-BSA (Sigma ${ }^{\circledR}$ ), fluorescein isothiocyanate isomer (FITC; Sigma ${ }^{\circledR}$ ), and FITC-BSA $\left(\right.$ Sigma $\left.^{\circledR}\right)$. In order to verify the 
electric charges of the four different fluorophores, electrophoresis was carried out. Since the electrophoresis places high charge difference at the opposing ends through the electrodes, the molecules between the two electrodes should migrate one direction or the other according to its charge. Rhodamine-BSA and Rhodamine $6 \mathrm{G}$ migrated toward the negatively charged electrode, which verified that these two fluorophores are positively charged. The FITC and FITC-BSA migrated toward the positively charged electrode, which verified that these fluorophores are negatively charged in Fig 2.

Bovine serum albumin (BSA) was conjugated to each fluorophore in rhodamine-BSA and FITC-BSA. The molecular weight of BSA is $66,463 \mathrm{Da}$, and its size is $140 \times 40 \times 40 \AA$ [22]. We used BSA-conjugated FITC to verify our FRAP system. The diffusivity of FITC-BSA in $2 \%$ agarose gel is $64 \pm 4 \mu \mathrm{m}^{2} / \mathrm{s}$ [23]. Hence, we could conclude that our system could generate reproducible and reliable data if we could obtain a similar result. Rhodamine-BSA was used to compare it with FITC-BSA because both were conjugated with BSA but had opposite polarities. Thus, the blocking effect of poly-DADMAC would be observable by comparing the diffusion coefficients of both materials. Fluorophores were conjugated to amino acids in BSA, which consists of 607 amino acids.

\section{Fluorescence Recovery After Photobleaching}

Fluorescence Recovery After Photobleaching (FRAP) is an optical technique that is used to quantify the two dimensional lateral diffusion of fluorescently labeled molecules. If a region of interest (ROI) is bleached by a high-intensity laser, molecules in the ROI are replaced by nonbleached molecules that are located outside of the ROI because of diffusion that is a result of the Brownian motion of the molecules [24]. First, the intensity of the 
fluorescently labeled sample is measured by using a low-power beam. Next, the fluorescently labeled molecules are bleached quickly by a high-power beam. The ROI diameter is usually selected to be between $1 \mu \mathrm{m}$ and $100 \mu \mathrm{m}$; diameter of $80 \mu \mathrm{m}$ was used in this experiment. After photobleaching, diffusion from the other regions to the ROI is monitored by using the low-power beam. The intensity recovery curve of the photobleached area is fitted to a suitable FRAP model from which the diffusion coefficient D of the sample molecules can be determined. The light intensity of the photobleached area cannot be exactly same as that of the unbleached area because some parts of the bleached area are not replaced by intact fluorophores. Thus, it is little lower than the initial intensity [25-27]. If the light exhibits a Gaussian profile, then the diffusion equation for the molecules can be determined according to the following three theories.

The first theory is the single exponential method:

$$
I(t)=I(\infty)\left(1-e^{-\tau_{d} t}\right)
$$

This method supposes that the intensity recovery curve follows an exponential curve. $\mathrm{I}(\mathrm{t})$ is the intensity of the ROI that has been normalized to the region that was not influenced by the bleaching because it was far enough from the ROI. $\mathrm{I}(\infty)$ is the intensity of the plateau level. $\tau_{d}$, which is called the characteristic diffusion time or half maximum time. It is the time over which the intensity of the ROI recovers to half of $\mathrm{I}(\infty)$. The diffusion coefficient could be calculated by using the following equation [28]:

$$
\mathrm{D}=\frac{\omega^{2}}{\pi \tau_{d}}
$$

Where $\mathrm{D}$ is the diffusion coefficient and $\omega$ is the diameter of the ROI. The second method is the Soumpasis diffusion fitting method [29]: 


$$
\frac{I(t)-I(0)}{I(\infty)-I(0)}=e^{-\tau_{d}(2 t)^{-1}}\left[B_{0}\left(\tau_{d}(2 t)^{-1}\right)+B_{1}\left(\tau_{d}(2 t)^{-1}\right)\right](3)
$$

The Soumpasis method tries to determine the exact diffusion coefficient by assuming that the intensity recovery curve can be expressed by two equations rather than a single equation. However, the two equations originate from a theoretical prediction and exhibit errors when they are applied to real experimental data. To solve this problem, Ellenberg et al. introduced an empirical equation based on his experiments [30]:

$$
\mathrm{I}(\mathrm{t})=\mathrm{I}(\infty)\left[1-\left(\frac{\omega^{2}}{\omega^{2}+4 \pi D_{t}}\right)^{\frac{1}{2}}\right]
$$

Diffusion coefficient D can be represented by the following equation:

$$
\mathrm{D}=\frac{3 \omega^{2}}{4 \pi \tau_{d}}
$$

We employed Ellenberg's empirical equation to calculate the diffusion coefficient in our experiments.

We used a FV1000 (Olympus ${ }^{\circledR}$ ) and a FV-10-ASW (Olympus ${ }^{\circledR}$ ) to measure the changes in intensity of the samples and calculated the intensity of each ROI by using the Image $\mathrm{J}$ program. The measured intensity of each type of fluorescent molecule was saved as a text file and its respective diffusion coefficient was calculated by using the Igor Pro program.

\section{Long term diffusivity experiment}

The long term diffusivity experiment is an alternative method to measure the diffusion coefficients of fluorescent materials. Although FRAP can be used to measure the local diffusivity of a material, the long term diffusivity experiment can be used to measure realistic diffusion speeds in a microchannel. The PDMS microchannel is a fully open cavity from the 
inlet to the outlet. The Poly-DADMAC is loaded into the channel through the use of the capillary force. The photoinitiator added to the Poly-DADMAC allows it to be cured by UV irradiation at 10 Watts for 10 seconds. After UV curing, the channel will appear clogged with Poly-DADMAC, but the Poly-DADMAC has a porous structure through which the fluorophores can diffuse through. The $20 \mu \mathrm{L}$ of each of the four fluorophores are loaded onto the inlet of the PDMS channel. In order to maintain constant density of the materials at the inlet, a thermohygrostat was used. The materials were added to the inlet once every 24 hours to prevent dehydration, and the diffusion of the materials towards the outlet was observed. The fluorescence intensity of the microchannel at $2.5 \mathrm{~mm}$ intervals was measured every 2 days from day 1 by taking a photo using the microscope and the diffusion coefficient was calculated from the fluorescence intensity.

\section{Results}

FRAP

First, we measure the diffusivity of FITC-BSA and rhodamine-BSA in the $2 \%$ agarose gel to validate the accuracy of our FRAP system. Fig. 3 shows the recovery curve of FITC-BSA after bleaching.

The diffusivity of FITC-BSA was calculated to be $58 \pm 3 \mu \mathrm{m}^{2} / \mathrm{s}$. This was $8 \%$ in error when compared to the value of $64 \pm 4 \mu \mathrm{m}^{2} / \mathrm{s}$ that was obtained from a reference paper. The error may take place from specimen, microscope and a curve fitting method, etc., but the $8 \%$ error is acceptable for measuring the diffusion coefficient in liquid phase [31]. The diffusivity of rhodamine-BSA was $60 \pm 3 \mu \mathrm{m}^{2} / \mathrm{s}$, which was close to that of FITC-BSA. This result was 
reasonable because agarose gel is a nonpolar material and the error rate with respect to the reference value was 5\%. Thus, we concluded that our FRAP system provided valid results.

Next, we measured the diffusivity of rhodamine 6G, FITC-BSA, and FITC in the polyDADMAC by using the same conditions as for the previous experiment. For the case of negatively charged Rhodamine and Rhodamine-BSA, their charge causes slow diffusion within the positively charged ion exchange resin, poly-DADMAC. Additionally, the FRAP test came out to be inconclusive for Rhodamine-BSA due to the lack of recovery after bleaching step. Although Rhodamine did recover after bleaching, increased mass and size of Rhodamine-BSA due to the conjugation of BSA seems to have lowered recovery rate Rhodamine-BSA far below the measurable amount.

Fig. 4 shows the graphs of recovered intensities of the fluorophores in the ROI after photobleaching. The values were normalized and therefore the initial intensities are unity. Using the graph, the diffusivity of each fluorophore in poly-DADMAC was calculated (Table 1).

The diffusivities of rhodamine $6 \mathrm{G}$ and rhodamine-BSA were much smaller than those of FITC and FITC-BSA. This was because rhodamine has a positive charge while FITC has a negative charge. Also, FITC-BSA diffused more slowly than FITC because its molecular weight was higher than FITC due to the conjugated BSA. Rhodamine $6 \mathrm{G}$ diffused the most slowly and FITC was the fastest in poly-DADMAC.

\section{Long term diffusivity experiment}

By carrying out the long term diffusivity experiment, we were able to obtain realistic diffusion speeds for the fluorophores in the microchannel. Fig. 5 shows fluorescence microscopy images of FITC-BSA as it diffused through the microchannel. The images were 
taken every two days. The light intensity at $2.5 \mathrm{~mm}$ intervals from the inlet was measured and the diffusivity was calculated by using this information. Fig. 5(a) shows that the fluorophores diffused faster on day 1 than the other days because of the concentration gradient. This meant that the ratio of the concentration to the distance between two points as well as the diffusion velocity were proportional to the concentration gradient. The concentration gradients in the early days were higher than those of the later days, and thus the diffusion velocity decreased during the experiment.

Fig. 6 shows the diffusion lengths of each fluorophore in the poly-DADMAC cured microchannel. The diffusion lengths of rhodamine 6G, FITC and FITC-BSA were same on day 11 because all of the molecules diffused to the end of the microchannel. The diffusion coefficient of each fluorophore that was calculated using the data from the long-term diffusivity experiment is shown in Table 2. The diffusion rate differences due to varying PDMS channel widths are indicated in the table 3. In this study, the diffusion rate differences are calculated as the obtained diffusion coefficients for different channel widths are compared with the average diffusion coefficient for each case. The increase in the average diffusion rate was observed as the channel width was increased. Additionally BSA conjugated fluorophore seem to have smaller differences.

Rhodamine-BSA was the slowest material with diffusion coefficient of $1.323 \mu \mathrm{m}^{2} / \mathrm{s}$, while FITC was the fastest material with a diffusion coefficient of $5.622 \mu \mathrm{m}^{2} / \mathrm{s}$. FITC was the fastest at diffusing followed by FITC-BSA, rhodamine 6G, and rhodamine-BSA for both FRAP and the long-term diffusivity experiment. The relative order of the diffusion coefficients were the same for both FRAP method and the long term diffusion tests. However, the calculated diffusion coefficients of the fluorophores were different between the 
FRAP and long term diffusion tests. The diffusion coefficients for FRAP were much smaller than those from the FRAP method.

\section{Discussion}

Diffusion coefficients of the four fluorophores in poly-DADMAC were measured by two methods in this study. When measured by FRAP, the diffusion coefficient of cationic rhodamine $6 \mathrm{G}$ was $1.179 \mu \mathrm{m}^{2} / \mathrm{s}$. This was much smaller value than that of FITC and FITCBSA, which were $9.206 \mu \mathrm{m}^{2} / \mathrm{s}$ and $3.721 \mu \mathrm{m}^{2} / \mathrm{s}$, respectively. This shows that molecules with positive charges diffuse slower than those with negative-charges in the cationic hydrogel poly-DADMAC. We were not able to measure the diffusion coefficient of rhodamine-BSA by FRAP because the bleached area did not recover.

To verify our results, a long-term diffusivity test was conducted and the results were compared to those for FRAP. The diffusion coefficients of rhodamine 6G, rhodamine-BSA, FITC, and FITC-BSA when measured by the long-term diffusivity test were $4.629 \mu \mathrm{m}^{2} / \mathrm{s}$, $1.323 \mu \mathrm{m}^{2} / \mathrm{s}, 5.622 \mu \mathrm{m}^{2} / \mathrm{s}$, and $5.291 \mu \mathrm{m}^{2} / \mathrm{s}$, respectively. The relative order of diffusivity was the same by both methods but the absolute values for the diffusivities were different. The experimental difference might have occurred due to the locally non-uniform porosity of $3 \mathrm{D}$ hydrogels. The effect of the non-uniform porosity of 3D hydrogels is smaller in the FRAP method due to the radial diffusion of the fluorophores, but greater in the long-term diffusion test due to its linear diffusion mechanism. In addition, another difference was that the diffusion velocity decreased as time progressed by long-term diffusivity test method. Although the different diffusion coefficients are obtained from the two methods, the orders are identical. In addition, we can take the values of the diffusion coefficient from the FRAP 
rather than the long-term diffusivity test if we consider the local diffusion coefficients without considering the uniformity of hydrogels.

Diffusion coefficients for FITC and FITC-BSA were not much different from each other as seen by the long-term diffusivity test. Therefore, the pore size of poly-DADMAC should be much larger than the size of BSA. Poly-DADMAC has pores interspersed in a uniform structure and thus the fluorophores were affected when they travelled through the pores. FITC and FITC-BSA were not influenced very much by the cation because they had negative charges. However, the diffusion coefficient of rhodamine-BSA was much smaller than that of rhodamine $6 \mathrm{G}$. Rhodamine $6 \mathrm{G}$ is smaller than rhodamine-BSA and thus the cations in rhodamine-BSA might have been influenced more than those in rhodamine $6 \mathrm{G}$ when the molecule passed through the pores in the poly-DADMAC. FITC-BSA seemed to be able to pass through the pores of poly-DADMAC faster than rhodamine-BSA because FITC has negative charge.

\section{Conclusions}

In this paper, the diffusion coefficients of biomaterials with different charge conditions in poly-DADMAC were measured to study the effect of the cationic poly-DADMAC as a 3D hydrogel. FRAP and a long-term measurement method that involved using a microchannel were employed to measure and compare the diffusion coefficient of each fluorophore. We demonstrated that the diffusion coefficients of the cations were smaller than those of the anions in poly-DADMAC because of the cations that were trapped in poly-DADMAC. 


\section{Acknowledgements}

This research was supported by the convergence technology development program for bionic arm through the National Research Foundation of Korea(NRF) funded by the Ministry of Science \& ICT (No. 2017M3C1B2085292)

\section{References}

[1] Park K, Shalaby SW, Park H (1993) Biodegradable hydrogels for drug delivery (Co. Lancaster, U.K.) 6:2

[2] Omidian H, Rocca JG, Park KN (2005) Advences in superporous hydrogels. J Control Release 102:3-12

[3] Appelmana TP, Mizrahi J, Elisseeff JH, Seliktar D (2011) The influence of biological motifs and dynamic mechanical stimulation in hydrogel scaffold systems on the phenotype of chondrocytes. Biomaterials 32:1508-1516.

[4] Leipziga ND, Wyliec RG, Kim H, Shoichet MS (2011) Differentiation of neural stem cells in three-dimensional growth factor-immobilized chitosan hydrogel scaffolds. Biomaterials 32:57-64

[5] Yang W, Xue H, Carra LR, Wang J, Jiang S (2011) Zwitterionic poly(carboxybetaine) hydrogels for glucose biosensors in complex media. Biosens Bioelectron 26:2454-2459

[6] Gupta P, Vermani K, Garg S (2002) Hydrogels: from controlles release to pH-responsive drug delivery. Drug Discov Today 7:569-579

[7] Hoarea TR Kohaneb DS (2008) Hydrogels in drug delivery: Progress and challenges. Polymer 49:1993-2007 
[8] Pallabi D, Deeptak B, Sudipto R (2012) An experimental study of the swelling behaviour of spherical hydrogel in water and the formulation of a theoretical model for its explanation. Int J Drug Delivery 4:20-30

[9] Li Y, Maciel D, Tomas H, Rodrigues J, Ma H, Shi H (2011) pH sensitive Laponite/alginate hybrid hydrogels: swelling behaviour and release mechanism, Soft Matter 7: $6231-6238$

[10] Ye F, Yaghmur A, Jensen H, Larsen SW, Larsen C, Østergaard J (2011) Real-time UV imaging of drug diffusion and release from Pluronic F127 hydrogels. Eur J Pharm Sci $43: 236-243$

[11] Yılmaz Y, Gelir A, Salehli F, Nigmatullin RR, Arbuzov AA (2006) Dielectric study of neutral and charged hydrogels during the swelling process. J Chem Phys 125:234705

[12] Lee PI (1985) Kinetics of drug release from hydrogel matrices. J Control Release 2:277288

[13] Stroe-Biezen S.A.M., Everaerts FM, Janssen LJJ, Tacken RA (1993) Difusion coefficients of oxygen, hydrogen peroxide and glucose in a hydrogel. Analytica Chimica Acta 273:553-560

[14] Li RH, Altreuter DH, Gentile FT (1996) Transport characterization of hydrogel matricies for cell encapsulation. Biotechnol Bioeng 50:365-373

[15] Matsuyama H, Teramoto M, Urano H (1997) Analysis of solute diffusion in poly(vinyl alcohol) hydrogel membrane. J Membr Sci 126:151-160 
[16] Koutsopoulos S, Unsworth LD, Nagai Y, Zhang S (2009) Controlled release of functional proteins through designer self-assembling peptide nanofilter hydrogel scaffold. PNAS 106:4623-4628

[17] Liang S, Xu J, Weng L, Dai H, Zhang X, Zhang L (2006) Protein diffusion in agarose hydrogel in situ measured by improved refractive index method. J Control Release 115:189196

[18] Park JH, Chung BG, Lee WG, Kim J, Brigham MD, Shim J, Lee S, Hwang CM, Durmus NG, Demirci U, Khademhosseini A (2010) Microporous cell-laden hydrogels for engineered tissue constructs. Biotechnol Bioeng 106:138-148

[19] Lopez-Sanchez P, Schuster E, Wang D, Gidley MJ, Strom A (2015) Diffusion of macromolecules in self-assembled cellulose/hemicellulose hydrogels. Soft Matter 11:40024010

[20] Patra S, Bal DK, Ganguly S (2015) Diffusion of moisture from hydrogel scaffold with induced porosity from self-assembled bubble. Drying Technol 33:336-345

[21] http://www.microchem.com/pdf/SU-82000DataSheet2025thru2075Ver4.pdf

[22] Wright AK, Thompson MR (1975) Hydrodynamic structure of bovine serum albumin determined by transient electric birefringence. Biophys J 15:137-141

[23] Pluen A, Netti PA, Jain RK, Berk DA (1999) Diffusion of macromolecules in agarose gels: comparison of linear and globular configurations. Biophys J 77:542-552 
[24] Goehring NW, Chowdhury D, Hyman AA, Grill SW (2010) FRAP analysis of membrane-associated proteins: lateral diffusion and membrane-cytoplasmic exchange. Biophys J 99:2443-2452

[25] Axelrod D, Koppel DE, Schlessinger J, Elson E, Webb WW (1976) Mobility measurement by analysis of fluorescence photobleaching recovery kinetics. Biophys $\mathrm{J}$ $16: 1055-1069$

[26] Peters R, Peters J, Tews KH, Bahr W (1974) A microfluorimetric study of translational diffusion in erythrocyte membranes. Biochim Biophys 367:282-294

[27] Braeckmans K, Buyens K, Naeye B, Vercauteren D, Deschout H, Raemdonck K, Remaut K, Sanders NN, Demeester J, De Smedt SC (2010) Advanced fluorescence microscopy methods illuminate the transfection pathway of nucleic acid nanoparticles. $\mathbf{J}$ Control Release 148:69-74

[28] Axelrod D, Koppel DE, Schlessinger J, Elson E, Webb WW (1976) Mobility measurement by analysis of fluorescence photobleaching recovery kinetics. Biophys $\mathrm{J}$ $16: 1055-1069$

[29] Soumpasis DM (1983) Theoretical analysis of fluorescence photobleaching recovery experiments. Biophys J 41:95-97

[30] Ellenberg J, Siggia ED, Moreira JE, Smith CL, Presley JF, Worman HJ, LippincottSchwartz JJ (1997) Nuclear membrane dynamics and reassembly in living cells: targeting of an inner nuclear membrane protein in interphase and mitosis. Cell Biol 138:1193-1206 [31] Milozic N, Lubej M, Novak P, Znidarsic-Plazl P, Plazl I (2014) Evaluation of diffusion coefficient determination using a microfluidic device. Chem. Biochem. Eng. Q. 28: 215-223 


\section{Tables}

Table 1. Diffusion coefficients in poly-DADMAC measured by FRAP

\begin{tabular}{|c|c|c|}
\hline & $\begin{array}{l}\text { Diffusivity } \\
\text { in } 2 \% \\
\text { agarose gel } \\
\left(\mu \mathrm{m}^{2} / \mathrm{s}\right)\end{array}$ & $\begin{array}{c}\text { Diffusivity in } \\
\text { poly- } \\
\text { DADMAC } \\
\left(\mu \mathrm{m}^{2} / \mathrm{s}\right)\end{array}$ \\
\hline $\begin{array}{c}\text { Rhodamine } \\
6 \mathrm{G}\end{array}$ & 63.951 & 1.179 \\
\hline $\begin{array}{c}\text { Rhodamine- } \\
\text { BSA }\end{array}$ & 60.211 & - \\
\hline FITC & 65.126 & 9.206 \\
\hline FITC-BSA & 58.281 & 3.721 \\
\hline
\end{tabular}


Table 2. Diffusion coefficients in poly-DADMAC measured by the long-term experiment

\begin{tabular}{|c|c|}
\hline & $\begin{array}{c}\text { Diffusivity } \\
\left(\mu \mathrm{m}^{2} / \mathrm{s}\right)\end{array}$ \\
\hline Rhodamine 6G & 4.629 \\
\hline Rhodamine- & 1.323 \\
\hline FITC & 5.622 \\
\hline FITC-BSA & 5.291 \\
\hline
\end{tabular}

Table 3. Effect of varying microchannel widths on the long term diffusion test

\begin{tabular}{|c|c|c|c|c|}
\hline Channel size & $\begin{array}{c}\text { rhodamine } \\
6 G\end{array}$ & $\begin{array}{c}\text { rhodamine- } \\
\text { BSA }\end{array}$ & FITC & FITC-BSA \\
\hline 200um & $-3.9 \%$ & $-1.4 \%$ & $-2.2 \%$ & $-2.7 \%$ \\
\hline $400 \mathrm{um}$ & $-2.1 \%$ & $-0.6 \%$ & $+0.2 \%$ & $+0.4 \%$ \\
\hline $600 \mathrm{um}$ & $+1.4 \%$ & $+2.0 \%$ & $+3.9 \%$ & $+2.6 \%$ \\
\hline $800 \mathrm{um}$ & $+3.8 \%$ & $+2.5 \%$ & $+5.1 \%$ & $+4.7 \%$ \\
\hline 1000um & $+4.2 \%$ & $+2.7 \%$ & $+6 \%$ & $+5.3 \%$ \\
\hline
\end{tabular}




\section{Figure Captions}

Fig. 1. Schematic and photos of the fabricated PDMS microchannel

Fig. 2. Fluorescence images after electrophoresis of the fluorophores. The red line in the middle is the start line. Rhodamine-BSA and rhodamine 6G moved to the cathode because they had negative charges while FITC-BSA and FITC had positive charges and thus moved to the anode.

Fig. 3. FITC-BSA FRAP data for $2 \%$ agarose

Fig. 4. Intensity changes of rhodamine, FITC, and FITC-BSA in the poly-DADMAC in the bleached area.

Fig. 5. (a-f) Fluorescence images of FITC-BSA diffusion in poly-DADMAC in the microchannel on (a) day 1, (b) day 3, (c) day 5, (d) day 7, (e) day 9, and (f) day 11

Fig. 6. Diffusion lengths along the microchannel for each measurement. Rhodamine-BSA was the slowest at diffusing and FITC was the fastest. 
Figures

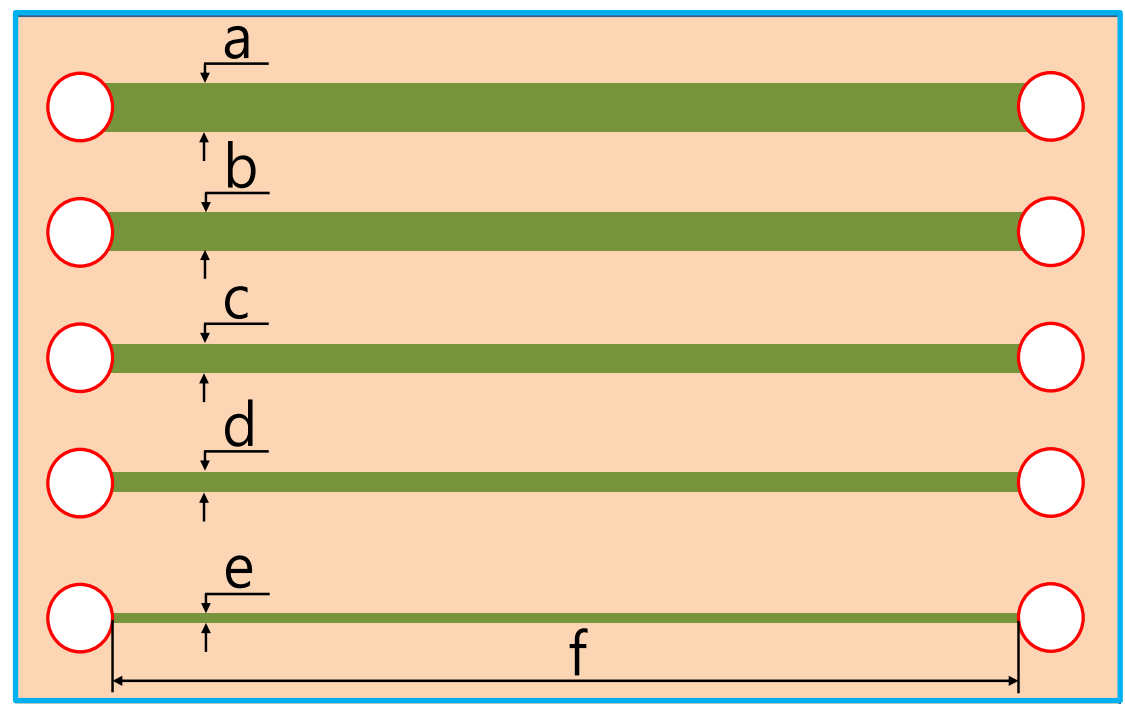

(a)

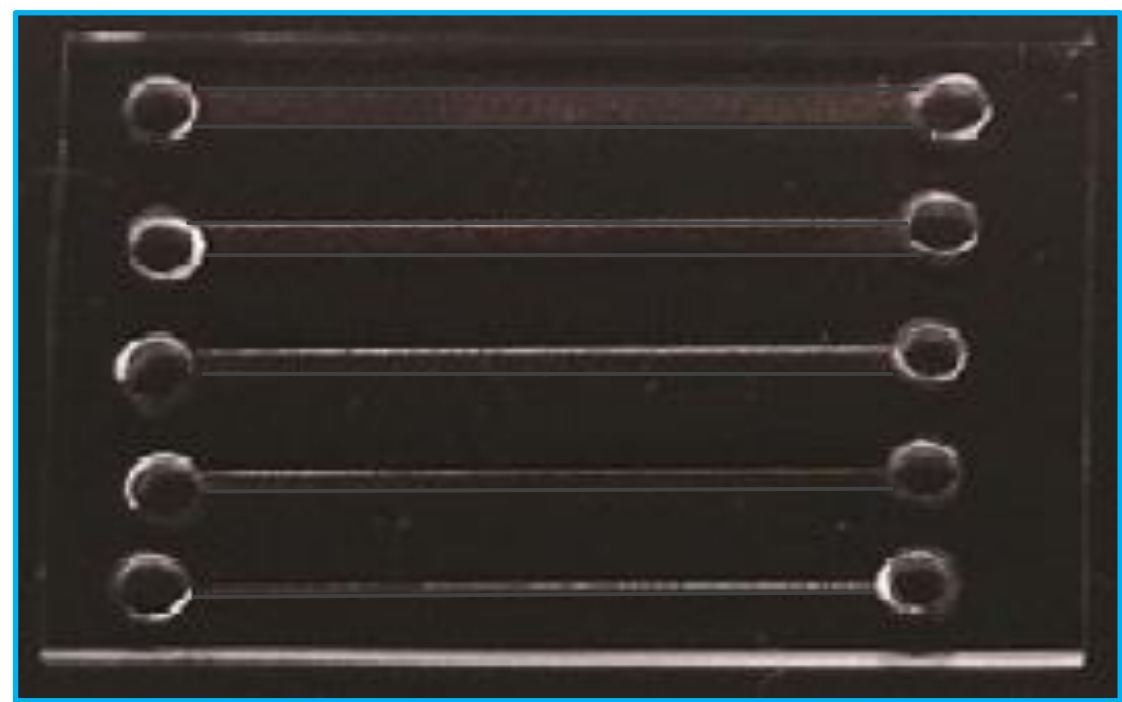

(b) 
Fig. 1. Schematic (a) and photos (b) of the fabricated PDMS microchannel: a,b,c,d,e and $f$ are $1000 \mu \mathrm{m}, 800 \mu \mathrm{m}, 600 \mu \mathrm{m}, 400 \mu \mathrm{m}, 200 \mu \mathrm{m}$ and $2.2 \mathrm{~cm}$, respectively. 


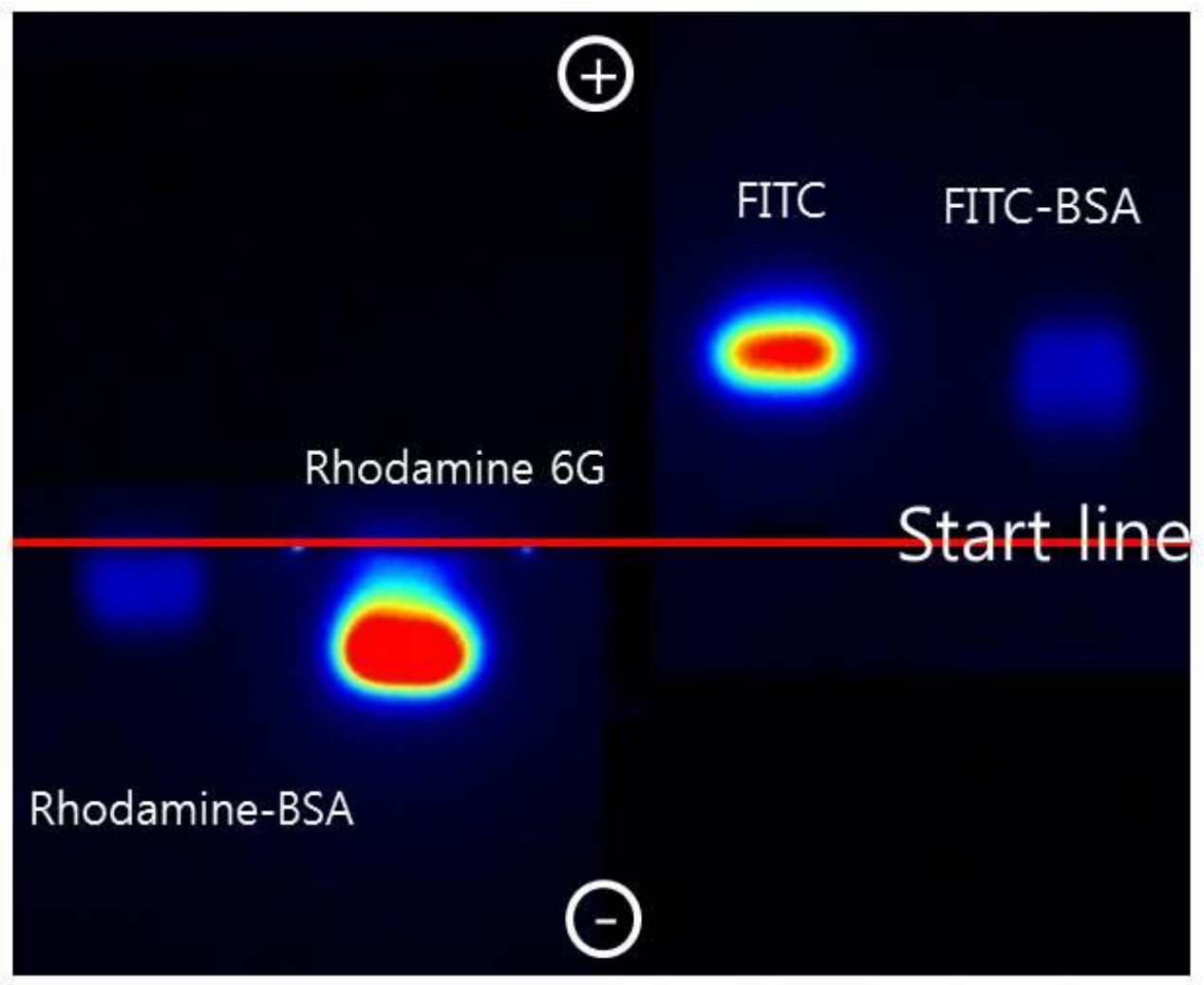

Fig. 2. Fluorescence images after electrophoresis of the fluorophores. The red line in the middle is the start line. Rhodamine-BSA and rhodamine $6 \mathrm{G}$ moved to the cathode because they had positive charges while FITC-BSA and FITC had negative charges and thus moved to the anode. 


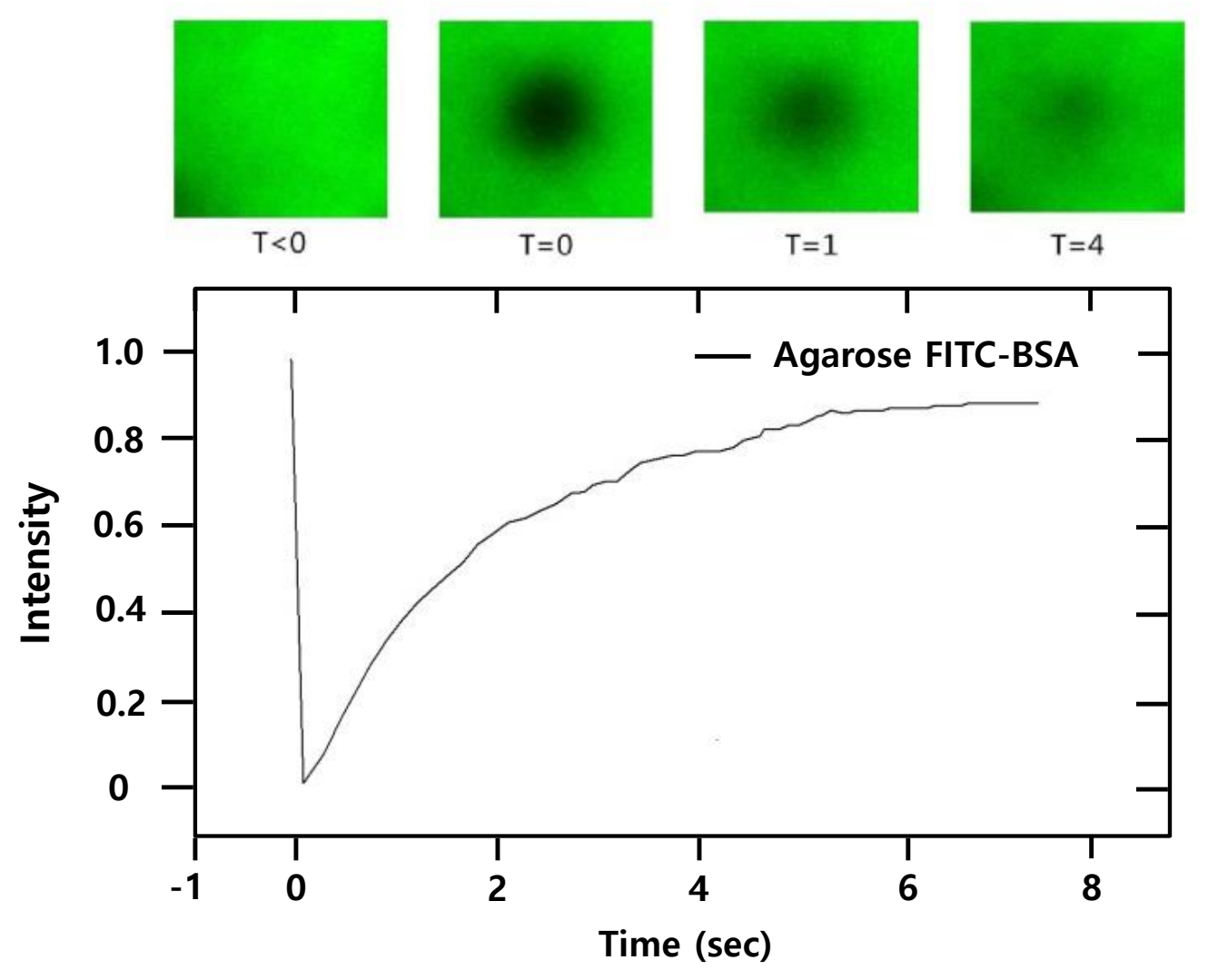

Fig. 3. FITC-BSA FRAP data for $2 \%$ agarose 


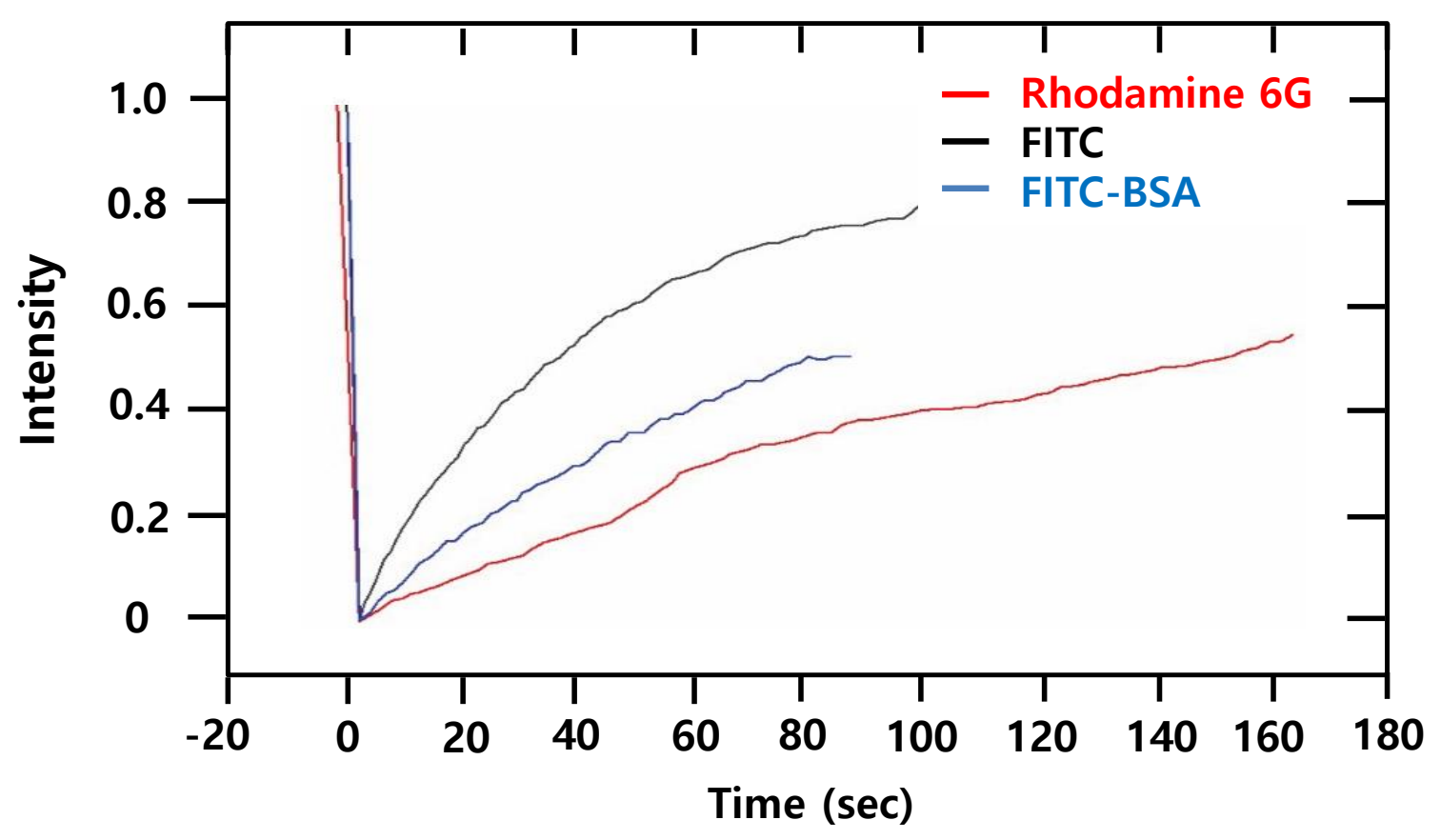

Fig. 4. Intensity changes of rhodamine, FITC, and FITC-BSA in the poly-DADMAC in the bleached area. 


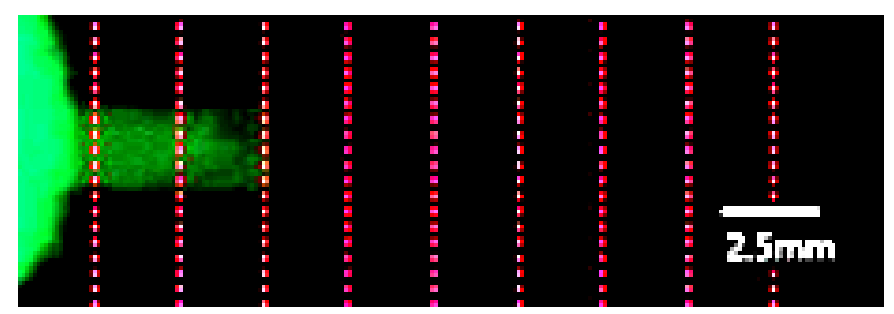

(a)

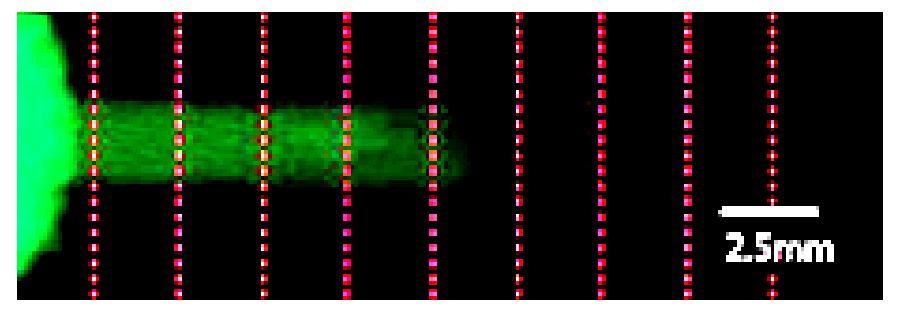

(b)

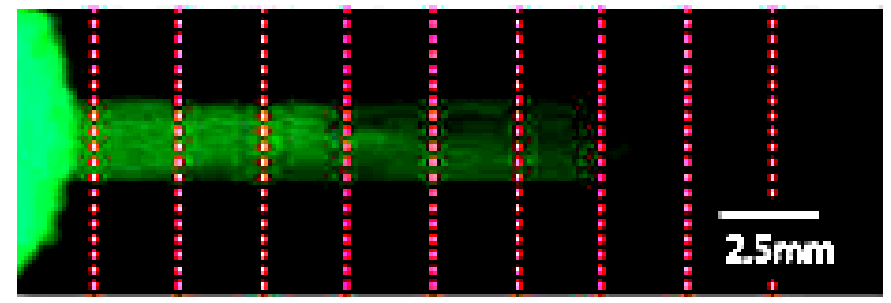

(c)

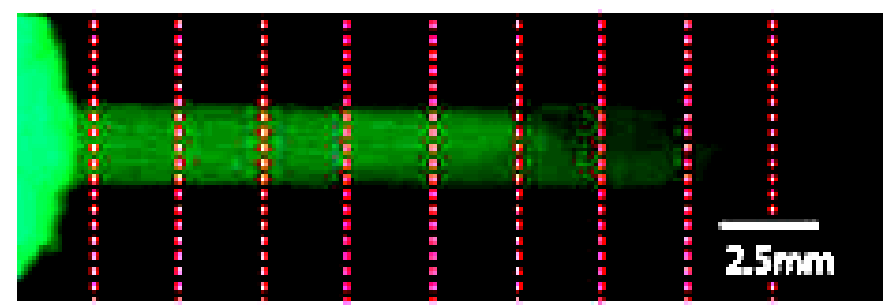

(d)

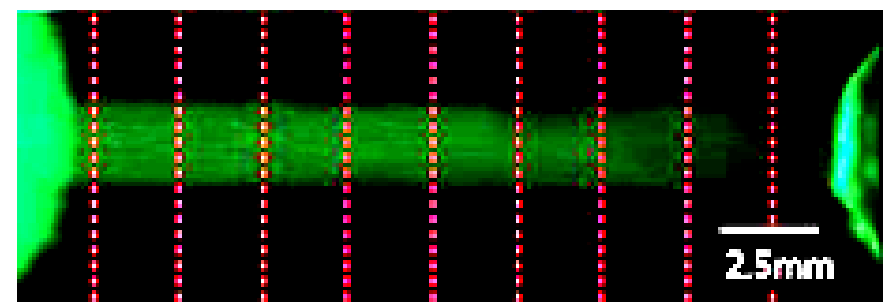

(e)

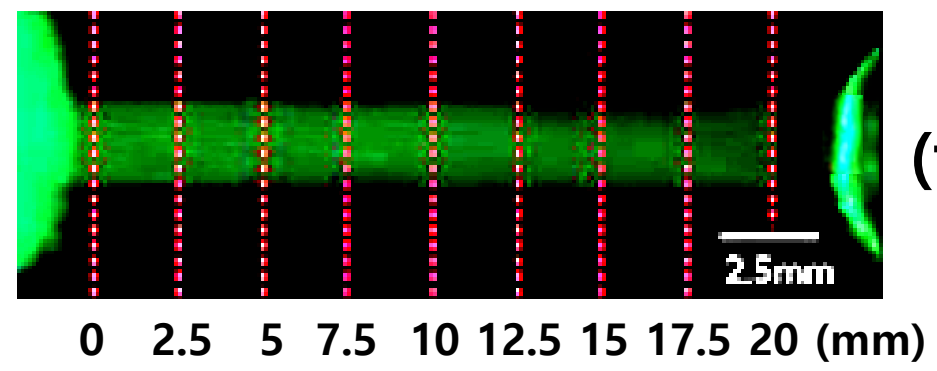

(f)

Fig. 5. (a-f) Fluorescence images of FITC-BSA diffusion in poly-DADMAC in the microchannel on (a) day 1, (b) day 3, (c) day 5, (d) day 7, (e) day 9, and (f) day 11 


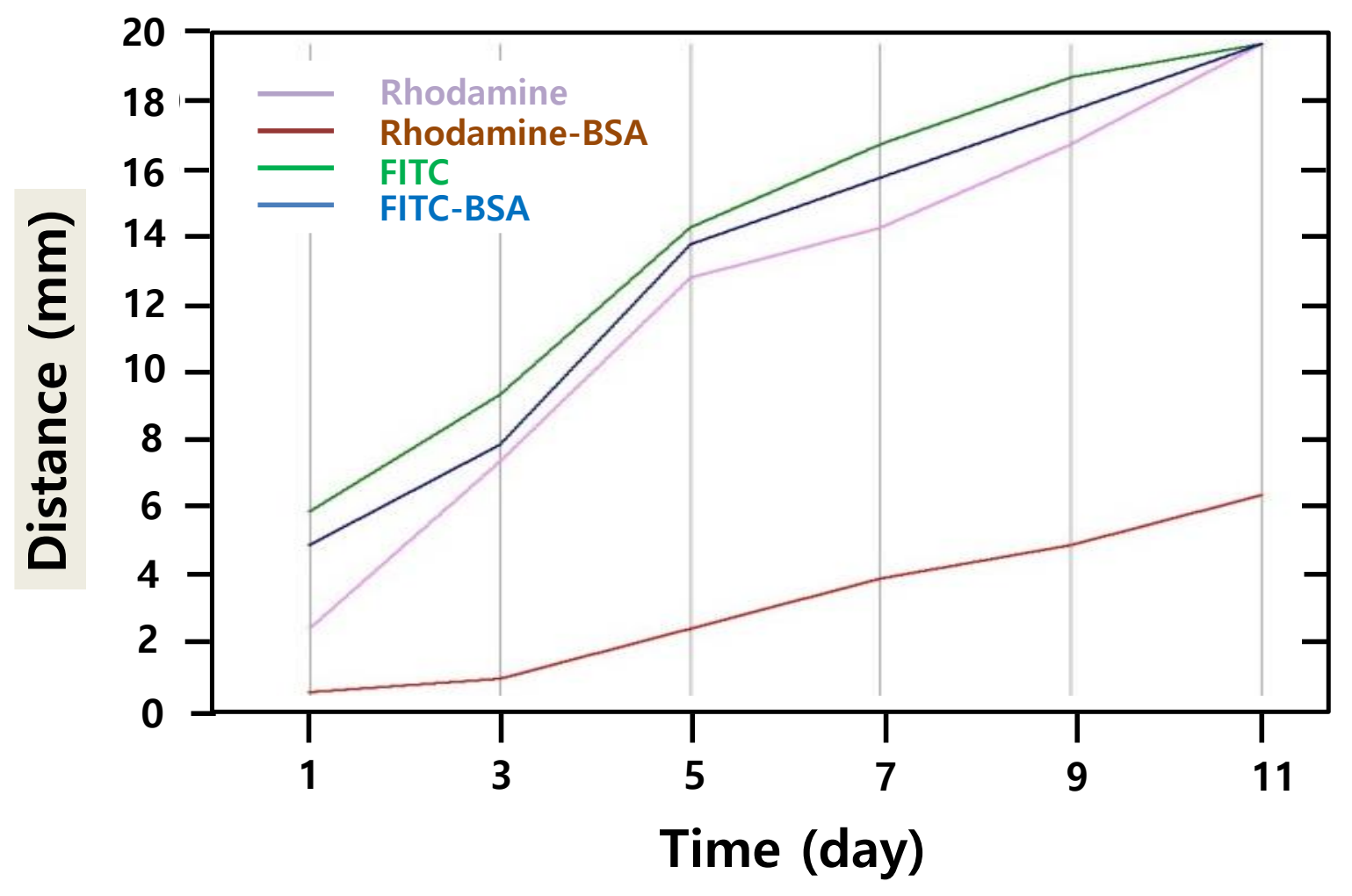

Fig. 6. Diffusion lengths along the microchannel for each measurement. Rhodamine-BSA was the slowest at diffusing and FITC was the fastest. 